\title{
Vibration Isolation Mechanism of Concrete Piles for Rayleigh Waves on Sand Foundations
}

\author{
Jinglei Liu $\mathbb{D}^{1},{ }^{1}$ Guishuai Feng $\mathbb{D}^{1},{ }^{1}$ Jian Zhang $\mathbb{D}^{1},{ }^{1}$ Xiaoyu Zhao $\mathbb{D}^{1},{ }^{1}$ Chuanqing Yu $\mathbb{D}$, \\ and Min Zhao ${ }^{2}$ \\ ${ }^{1}$ Hebei Key Laboratory of Diagnosis, Reconstruction and Anti-Disaster of Civil, Zhangjiakou 075000, China \\ ${ }^{2}$ School of Foreign Languages, Hebei University of Architecture, Zhangjiakou 075000, China \\ Correspondence should be addressed to Jinglei Liu; kingbest_1118@163.com
}

Received 27 July 2018; Revised 8 October 2018; Accepted 14 November 2018; Published 12 December 2018

Academic Editor: Pedro A. Costa

Copyright ( $\odot 2018$ Jinglei Liu et al. This is an open access article distributed under the Creative Commons Attribution License, which permits unrestricted use, distribution, and reproduction in any medium, provided the original work is properly cited.

To study the propagation characteristics of Rayleigh waves and the isolation mechanism of a single-row of piles by isolation effects, in this paper we draw a two-dimensional contour map of $\zeta$ (normalized acceleration amplitude relative to a measure close at the vibration source) using a vibration test carried out on a sand foundation. In this experiment, we study, in addition to the free field and the single pile cases, settings with two and three piles. The result shows that the vibration caused by the point source in the free field excites Rayleigh waves in a radial direction along the surface of the foundation. Meanwhile, the vibrations of the points along the propagation path on the surface of the foundation are gradually weakened. There is a steady transition when the $\zeta$ drops to 0.6 and a placid decline when $\zeta$ decreases to less than 0.25 . The vibration-shielded region, the strengthened region, and the strengthened strips will appear on the surface of the foundation. The vibration-shielded region is located behind the piles, and the region presents a trumpet-shaped area that takes the pile as the vertex. Increasing the quantity of piles contributes to increasing the vibration isolation effect, not only that involving the degree of isolation but also for the area of the shielded area. The vibration-strengthened regions include the diffraction regions at the pile corners on both sides of the single-row of piles and the scattering region at the gaps of the piles. In addition, the composite regions are located among the vibration source and the scattering and diffraction-strengthened regions. Increasing the number of piles has little influence on the scattering and diffraction-strengthened regions but can significantly enhance the vibrations of the composite regions. In general, the vibration-strengthened strips are connected with the scattering-strengthened regions. However, in the test of a single pile, the pile is connected to the diffraction-strengthened regions near its two anterior angles.

\section{Introduction}

With the steady progress of China's economy, the transportation system has developed rapidly, especially the rail transit which has grown both in volume and speed. However, the ensuing vibration pollution has also become increasingly serious and has adversely affected human health, buildings stability, and the normal use and maintenance of sensitive equipment [1-3]. The issue of vibration pollution has become one of the seven major public hazards, accounting for approximately 30 percent of all public hazards [4]. It seems appropriate to understand the vibration pollution and how to mitigate its effects.
The main sources of ground vibrations are highways, railways, heavy industrial machinery, and construction activities [5]. The issue of vibration pollution is largely related to soil dynamics, and an associated method to effectively control vibration pollution is to set up a vibration isolation barrier in the foundation. The form of the barrier varies and can be divided into continuous and discontinuous barriers [6]. Continuous barriers commonly used in projects are open trenches and in-filled trenches; the corresponding discontinuous barriers mainly include discontinuous row wells and piles, and the number of rows is determined by the project requirements [7]. Although the wells and the open trenches are relatively effective [8], the depths of these 
barriers are generally shallow. Their use is therefore limited to a certain extent when considering some engineering problems, such as soil stability and secure but low-cost construction [9]. Relatively speaking, row piles not only have a good vibration isolation effect [10] but also have good adaptability to environmental conditions and design requirements. There are some advantages over other types of vibration isolation barriers.

Many scholars have conducted different degrees of research on the vibration isolation effects and mechanisms of row piles. Aviles and Sanchez-Sesma [11] proposed a theoretical study of a single-row of rigid pile serving as an isolation barrier to elastic waves. Parameters such as the pile diameter, pile length, and pile spacing were analysed. Woods et al. [12] analysed the vibration isolation effect of wells and row piles via holography technology and proposed that the vibration isolation effect is better when the diameter of the single discontinuous barrier is larger than $1 / 6$ of the shielded wavelength. In an investigation presented by Kattis et al. [13, 14], the pile length, burial depth, and overall width are the primary factors that affect the vibration isolation effect, and the pile spacing is significant. $\mathrm{Xu}$ [15] studied the passive isolation of moving loads using pile rows embedded in saturated soil. The vibration isolation effect of single-row of piles for low speed moving loads is considered to be better than those of the higher speed moving loads. Additionally, the optimal length of piles for higher speed moving loads is shorter than that for lower speed moving loads. Liu and Wang [16] analysed the isolation effect of the discontinuous pile-group barriers on plane $\mathrm{P}$ and SV waves within a broad frequency band. The results show that multiple rows of piles should be adopted for low-frequency waves, and a pile group with more than 3 rows will not lead to a significant improvement in the isolation effect for the high-frequency waves. Sun [17] proposed a theoretical methodology that can calculate the multiple scattering of plane $\mathrm{SH}$ waves for multirow tubular piles with arbitrarily arranged and sectional dimensions. Shi and Gao [1] and $\mathrm{Xu}$ et al. [18] studied the passive vibration isolation of single-row of piles in a distant field using a numerical analysis method, and the results were compared.

However, the vibration isolation effect of row piles is regarded as a simple research topic. Most of the studies focus on the comparison of vibration isolation effects and the analysis of their influencing factors. A deeper exploration of the isolation mechanisms associated with isolation effects is lacking. The vibration isolation problem of single-row of piles excited by point vibration sources on sand foundations has been undertaken in this paper, and the isolation mechanism of single-row of piles from a two-dimensional perspective is discussed, which enriches the research findings in the field of vibration isolation for single-row of piles.

\section{Correlation Theory, Test Condition, and Procedure}

In soil dynamics, the foundation is generally abstracted into elastic media in an ideal half-space. The longitudinal wave
(P) caused expansion and compression of the medium, and the transverse wave $(S)$ caused rotation of the medium in the soil. When the above waves propagate to the surface of the soil, a surface wave named the Rayleigh wave (R) is formed [19]. A Rayleigh wave propagates only along the surface of the soil and decays exponentially with increasing depth. The vibration waves are not completely cut off when obstructed by barriers, and some will still propagate to the rear area of the barrier in some way. The lower the vibration energy propagating behind the barrier, the better the isolation performance of the barrier.

Take the discontinuous isolation barrier that is applied for a point vibration source as an example. There are three different approaches for a wave to pass through an isolation barrier: diffraction [20], transmission [21], and scattering [22]. Moreover, the isolation barrier can also reflect the wave [23]. Four types of waves corresponding to the above approaches exist behind the barrier: diffraction waves, transmission waves, scattering waves, and reflection waves, as shown in Figure 1.

The research results of Miller and Pursey [24] show that the energy carried by a Rayleigh wave can reach up to $67 \%$ of the total energy (supplemented by the $\mathrm{P}$ wave $7 \%$ and $\mathrm{S}$ wave $26 \%$ ), and the Rayleigh wave has the largest amplitude of the vibrational waves under the vertical excitation over a half-space. In addition, a Rayleigh wave propagates on the surface of the foundation; thus, its impact on and significance to the project are remarkable. Therefore, the content of this paper is mainly concerned with Rayleigh waves.

2.1. Details of Test Equipment. The main equipment is a set of control systems named WS-Z30, which consists of a control cabinet (including a data acquisition controller, signal generator, signal amplifier, and ICP adapter), electromagnetic exciter, computer, and a few accelerometers. The field site and the instrument used in the experiment are shown in Figure 2. Simple and stable signals are sufficient to meet the test requirements. The source is stable; in other words, the waves with specific frequencies and types can be output steadily and continually. Moreover, considering the simplicity of the test operation, a $30 \mathrm{~Hz}$ sinusoidal wave is selected as the signal in the experiment. The pile is poured using concrete with a strength grade of C30 (the National Standard of China "Code for Design of Concrete Structures" (GB 50010-2010) stipulates: when the standard value of cube compressive strength of concrete goes to $30 \mathrm{~N} /$ $\mathrm{mm}^{2}$, its strength grade is expressed as C30), the shape of which is a square with a side length of $10 \mathrm{~cm}$, and the lengths of which are $30 \mathrm{~cm}, 40 \mathrm{~cm}$, and $50 \mathrm{~cm}$. There are 11 accelerometers applied in the test, one of which is used for reference keeping the relative position with the exciter and others are used for the flow test. The mass of each sensor is $28.5 \mathrm{~g}$, the charge sensitivity is $4 \mathrm{pc} / \mathrm{ms}^{-2}$, the frequency response is 0.2 to $8000 \mathrm{~Hz}$, and the measurement range is $50 \mathrm{~m} / \mathrm{s}^{2}$. The sampling frequency was set to 5000 times per second in the test, and the measurement time was 5 seconds. 


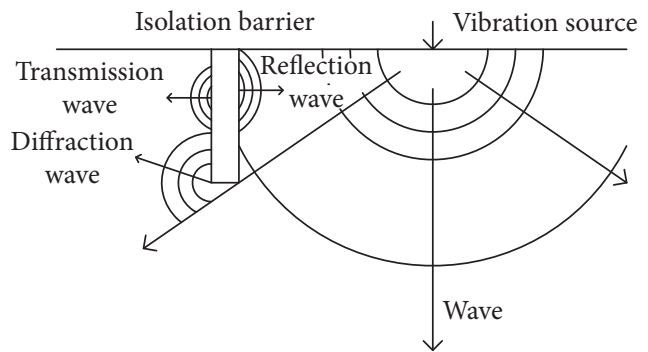

(a)

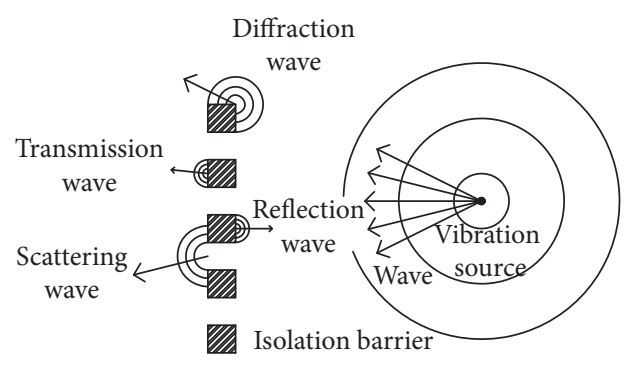

(b)

Figure 1: Types of the wave: (a) vertical view; (b) horizontal view.
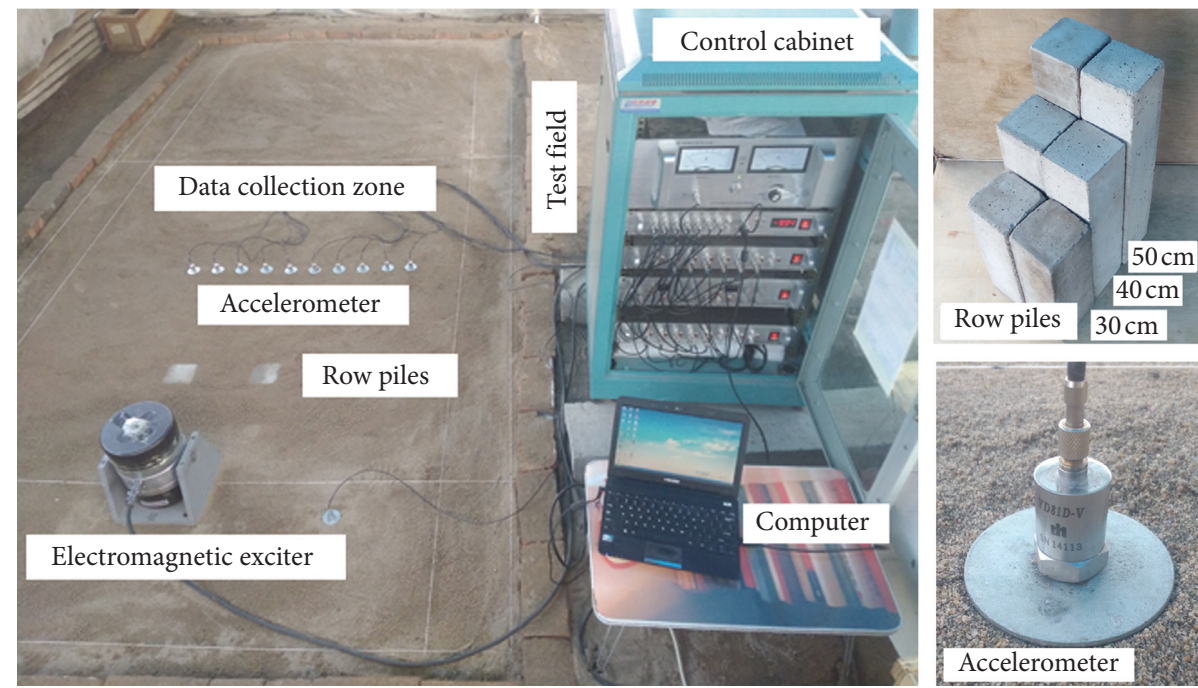

FIGURE 2: Details of the test instruments.

2.2. Geotechnical Setting of the Test Site. Note that the properties of sand are stable and the test variables are easy to control. Therefore, the field site is located in the suburbs, where artificial vibrations and other noises are infrequent. According to the borehole data, the soil layering of sand foundation is shown in Figure 3. The foundation is divided into two layers: the first layer is a medium sand layer with a depth of $10.81 \mathrm{~m}$ and the lower one is a coarse sand layer. The wave velocity of each soil layer is measured by single-hole layer-testing method, which is also listed in Figure 3. The wave velocity of medium sand layer is $110 \mathrm{~m} / \mathrm{s}$ and that of coarse sand layer is $202 \mathrm{~m} / \mathrm{s}$.

Field borehole samples show that a small amount of large gravel impurity is found in the medium sand layering. In order to eliminate the influence of the gravel impurity on the test, a pit with a plane size of $2 \mathrm{~m} \times 4 \mathrm{~m}$ and a depth of $5 \mathrm{~m}$ is excavated in sand foundation with certain supporting measures. After passing through the $5 \mathrm{~mm}$ aperture sieve, the sand excavated from the pit is rammed back into the pit. The method is used to eliminate the interference of the test site boundary and the impurities in the soil to the vibration wave and to improve the scientific nature of the test. The physical properties of the backfilled sand in the test site are shown in Table 1, the grain-size distribution curve of the sand in the field is shown in Figure 4, and the particle composition and related indexes are shown in Table 2.

2.3. Test Approach and General Procedure. The purpose of this paper is to discuss the isolation mechanism of singlerow of piles from a two-dimensional perspective. All the tests involved in this paper are carried out in the sand pit mentioned above, and the surface of the test site is leveled before each test. The plane size of the test site is $2 \mathrm{~m} \times 4 \mathrm{~m}$, and a rectangle-shaped data collection zone with the size of $1.6 \mathrm{~m} \times 2 \mathrm{~m}$ is located inside the field site. The exciter, concrete piles, and 357 test points are all in the zone. All the test points are evenly distributed in the data collection zone, and the spacing between each point and its surrounding points is $10 \mathrm{~cm}$. All points are numbered in sequence, and all numbers are continuous and unique. The test layouts are shown in Figure 5.

In order to reveal the essential characteristics of vibration isolation mechanism of single-row of concrete piles, the number of piles is limited to one, two, and three. Different pile numbers also corresponds to different positions, as shown in Figure 6. Combined with Figure 5, the exciter is located on the longitudinal axis of the data collection zone and the midpoint line of the row pile is parallel to the 


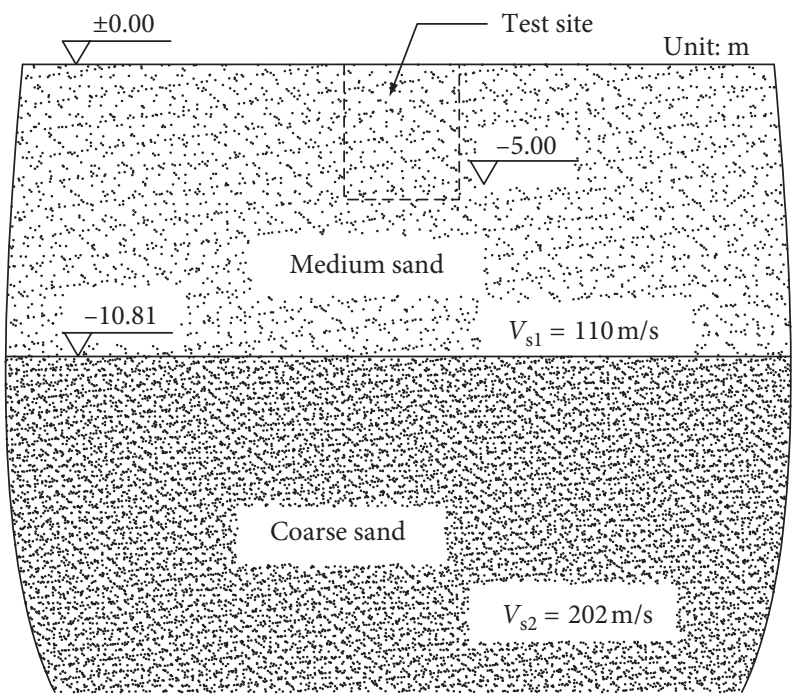

Figure 3: Distribution of soil layer in sand foundation.

TABLe 1: Physical index of the backfilled sand.

\begin{tabular}{lccccc}
\hline Index & Apparent density $\left(\mathrm{kg} \cdot \mathrm{m}^{-3}\right)$ & Bulk density $\left(\mathrm{kg} \cdot \mathrm{m}^{-3}\right)$ & Porosity $(\%)$ & Mud content $(\%)$ & Fineness modulus \\
\hline Value & 2632.6 & 1568 & 40.7 & 0.6 & 2.95 \\
\hline
\end{tabular}

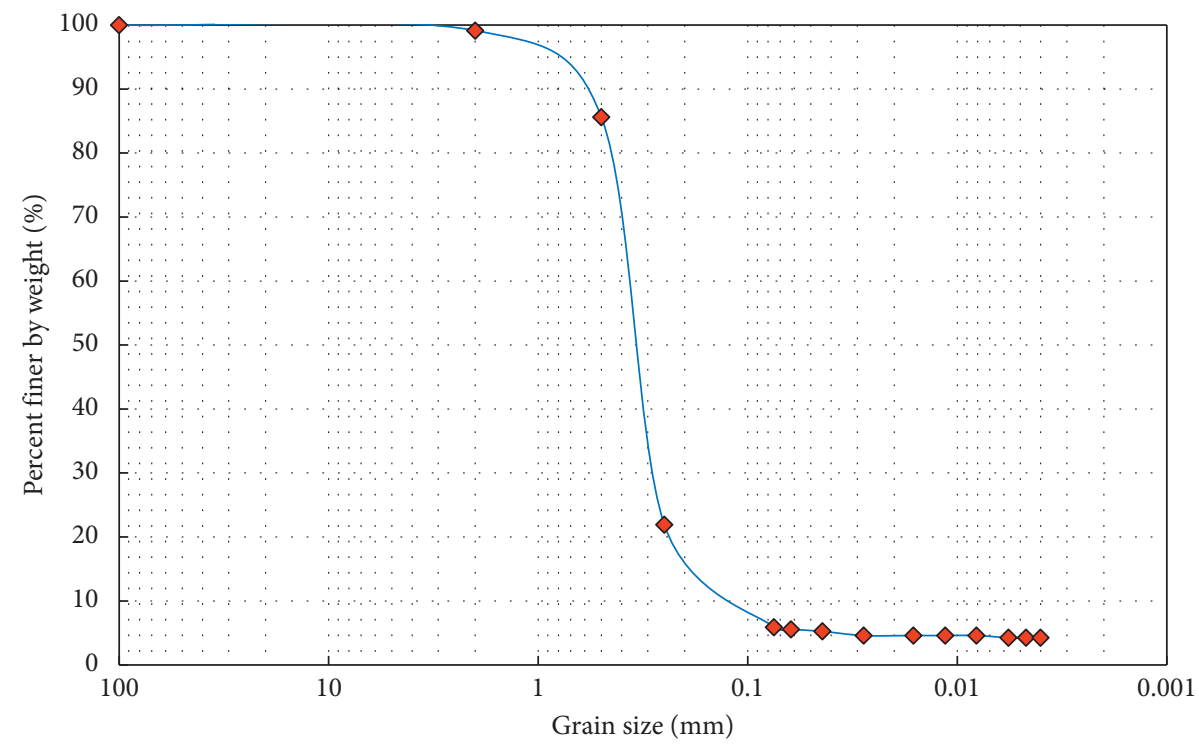

FIgURE 4: The grain-size distribution curve.

TABLE 2: Particle composition and related indexes of the backfilled sand.

\begin{tabular}{|c|c|c|c|c|c|c|c|c|c|c|c|}
\hline \multirow{3}{*}{ Particle composition } & \multicolumn{2}{|l|}{ Pebble } & \multicolumn{2}{|c|}{ Gravel } & \multicolumn{3}{|c|}{ Sand } & \multicolumn{3}{|c|}{ Silt } & \multirow[t]{2}{*}{ Clay } \\
\hline & & & & & & & & & & & \\
\hline & & 60 & 20 & 5 & 2 & 0.5 & 0.25 & 0.075 & 0.05 & 0.01 & 0.005 \\
\hline Particle size (mm) & $\sim$ & $\sim$ & $\sim$ & $\sim$ & $\sim$ & $\sim$ & $\sim$ & $\sim$ & $\sim$ & $\sim$ & $\sim$ \\
\hline & 60 & 20 & 5 & 2 & 0.5 & 0.25 & 0.075 & 0.05 & 0.01 & 0.005 & 0.002 \\
\hline Content (\%) & & & & 0.9 & 13.5 & 63.7 & 16.0 & 0.5 & 0.8 & 0.4 & $>0.0$ \\
\hline Particle composition index & & & & & & & & & & & \\
\hline Index & $d_{60}$ & & & $d_{30}$ & & & & & & & \\
\hline Value & 0.365 & & & 0.286 & & & & & & & \\
\hline
\end{tabular}




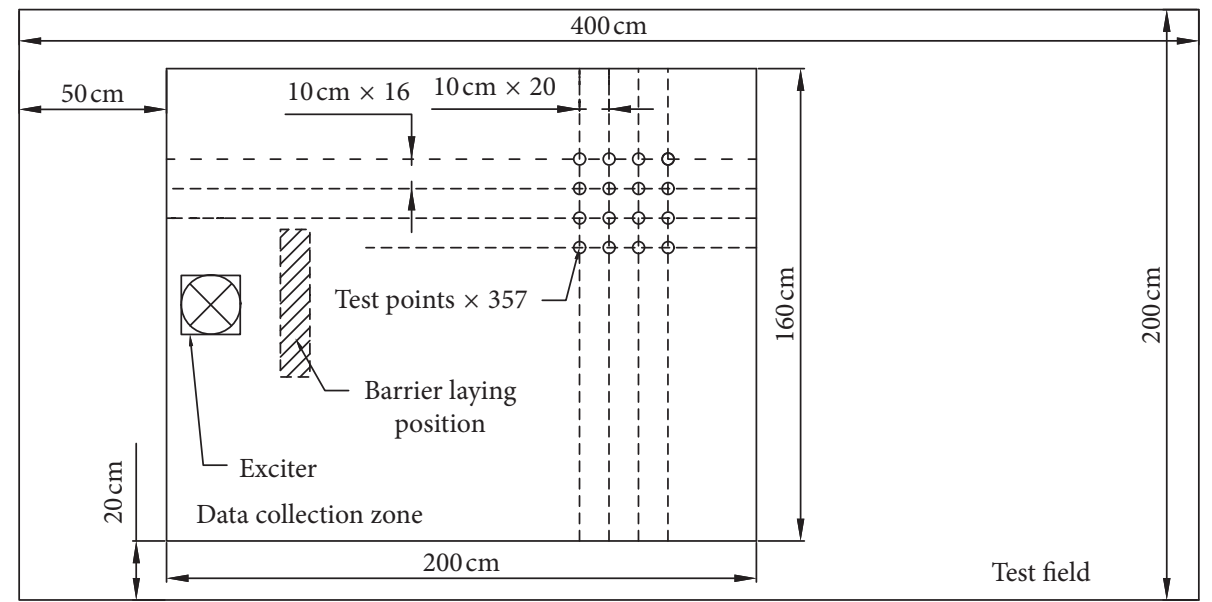

Figure 5: Test layouts.

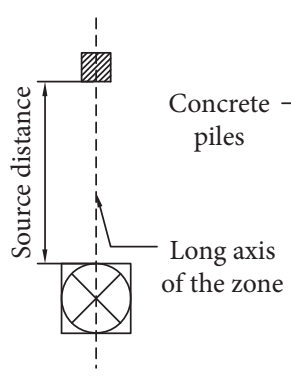

(a) (b)

(c)

FIgURE 6: Relative positions of the row pile and the exciter: (a) single pile; (b) double piles; (c) treble piles.

transverse axis. The difference lies in the fact that when the single pile and treble piles are set, the piles and the exciter are opposite and the piles pass through the longitudinal axis of the data collection zone, and when the double piles are set, the gap between the piles and the exciter are opposite and the gap passes through the longitudinal axis of the data collection zone. The purpose of setting up the contrast test is to compare double piles with single pile to investigate the influence of different positions between concrete piles and point vibration source and to compare treble piles with single pile to investigate the influence of the pile quantity.

In order to investigate the vibration on the surface of data collection zone under different pile quantities, 19 working conditions are designed in this paper as shown in Table 3 . The variables include the source distance $(L)$, the pile length $(H)$, and the clear distance $(D)$. The source distance $(L)$ denotes the distance between the exciter and the midpoint line of the row pile. The pile length $(H)$ denotes the length of a single pile. The clear distance $(D)$ denotes the distance between the exteriors of two adjacent piles (Figure 6). All working conditions can be integrated into 7 contrast test groups for comparative analysis, as shown in Table 4 . The test codes in the two tables are illustrated with the example of T2-343, as shown in Figure 7. Particularly, the free field refers to the natural foundation without any isolation barriers, so the test code of the free field in the
TABLE 3: The schedule working conditions.

\begin{tabular}{lcccc}
\hline Test code & Pile quantity & $L(\mathrm{~cm})$ & $H(\mathrm{~cm})$ & $D(\mathrm{~cm})$ \\
\hline T0 & 0 & $/$ & $/$ & $/$ \\
\hline T1-33 & & 30 & 30 & $/$ \\
T1-34 & 1 & 30 & 40 & $/$ \\
T1-35 & & 30 & 50 & $/$ \\
T1-24 & 20 & 40 & $/$ \\
T1-44 & & 40 & 40 & $/$ \\
\hline T3-341 & & 30 & 40 & 10 \\
T3-342 & 3 & 30 & 40 & 20 \\
T3-343 & & 30 & 40 & 30 \\
T3-333 & & 30 & 30 & 30 \\
T3-353 & & 20 & 50 & 30 \\
T3-243 & & 40 & 40 & 30 \\
T3-443 & & 30 & 40 & 30 \\
\hline T2-341 & & 30 & 40 & 10 \\
T2-342 & & 30 & 40 & 20 \\
T2-343 & & 30 & 30 & 30 \\
T2-333 & 2 & 30 & 50 & 30 \\
T2-353 & & 20 & 40 & 30 \\
T2-243 & & 40 & 40 & 30 \\
T2-443 & & & &
\end{tabular}

TABLE 4: The group of contrast test.

\begin{tabular}{lccc}
\hline Number & \multicolumn{3}{c}{ Detail of the contrast test } \\
\hline 1 & T1-33 & T3-333 & T2-333 \\
2 & T1-34 & T3-341 & T2-341 \\
3 & T1-34 & T3-342 & T2-342 \\
4 & T1-34 & T3-343 & T2-343 \\
5 & T1-35 & T3-353 & T2-353 \\
6 & T1-24 & T3-243 & T2-243 \\
7 & T1-44 & T3-443 & T2-443 \\
\hline
\end{tabular}

vibration isolation test is T0. The clear distance does not exist in the case of a single pile, which is not shown on the test code, such as in the case of T1-35.

The dissipation ratio of the acceleration amplitude represented by $\zeta$ is introduced to measure the relative strength of vibration, whose definition is shown in Equation (1): 


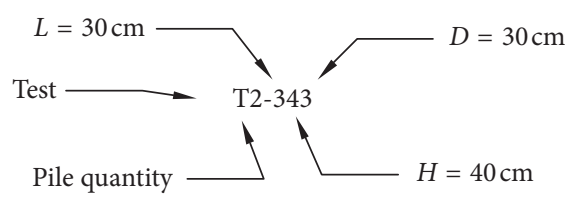

FIgURE 7: Illustration of the test code.

$$
\zeta=\frac{A^{*}}{A_{0}}
$$

where $A^{*}$ denotes the peak amplitude of the time history curve of the acceleration at each test point and $A_{0}$ denotes the corresponding peaks at the vibration source. Logically, $\zeta$ has the following properties: (a) $\zeta>0$; (b) at the vibration source, $\zeta=1$, and the infinite distance is close to $0 ;(\mathrm{c}) \zeta>1$ indicates the enhancement of vibration, and $\zeta<1$ indicates the opposite; and (d) $\zeta$ is essentially a relative value and not an absolute one. According to the values of $\zeta$ at each test point in the data collection zone, a $2 \mathrm{D}$ contour map of $\zeta$ can be drawn, which can be used to analyse the mechanism of concrete piles acting on Rayleigh waves.

With regard to the use of sensors: as the number of sensors is far less than the number of test points, all flow sensors are used to collect the vibration data from all points according to the sequence of the point number multiple times; to reduce the error, the reference sensor is set to the lateral rear of the exciter to collect the reference acceleration for each time point of the test, and it always keeps a fixed relative position with the exciter.

The principles for determining the values of $A^{*}$ at each test point are as follows: the ratio of the peak amplitude of the acceleration time history curve to the reference is taken as the value of $A^{*}$ at some point. The calculation principle of $A_{0}$ is as follows: the average value of $A^{*}$ of each test point, which is adjacent to both the sides and back of the exciter, is the value of $A_{0}$. The direct acquisition of data via the exciter cannot be realized, so an indirect method is used to calculate $A_{0}$ in the test.

Tests and data analysis are carried out in accordance with the following operating procedures:

(1) According to the requirements of the working conditions, take the concrete piles embedded in the predetermined position and the vibration exciter and the reference sensor put in place.

(2) Level the surface of the test site and mark the locations of the test points, the points occupied by the exciter and the piles need not be tested.

(3) The flow sensors are arranged on the $1^{\#}-10^{\#}$ test point in turn, input the sinusoidal wave signal with the frequency of $30 \mathrm{~Hz}$, the exciter vibrates for $5 \mathrm{~s}$, and 11 sensors receive 11 sets of vibration data.

(4) Calculate the ratio of the peak amplitude of the time history curve of the acceleration at each test point to the reference point; $A^{*}$ of $1^{\#}-10^{\#}$ can be obtained.

(5) Repeat step (3) and step (4) until all test points are completed.

(6) Calculate the average value of $A^{*}$ of each test point adjacent to both the sides and back of the exciter, $A_{0}$ can be obtained.
(7) Calculate the ratio of $A^{*}$ to $A_{0}$ of each measuring point; $\zeta$ of each test point can be obtained. If a certain drawing proportion is taken, the $2 \mathrm{D}$ contour map of $\zeta$ can be obtained.

\section{Results and Analysis}

The two-dimensional contour map of $\zeta$ is used to analyse the propagation characteristics of the Rayleigh wave on the surface of the sand foundation and the isolation mechanism of the single-row of piles. The scale of the map is $1: 10$, and the drawing area involves the entire data collection zone of the field site. The legend consists of 16 numerical sections with intervals of 0.1 . The propagation characteristics of the Rayleigh wave are mainly reflected in the test of the free field, which serves as a control to the row pile when researching the isolation mechanism. Taking into account the page space, reasonable selection, and necessary analysis, the tests of T0, T1-35, T3-353, and T2-353 are highlighted in Table 3 and are taken as representative samples for a detailed discussion. The rest are used only for reference, and no result of these cases is given in detail in this paper.

3.1. The Test of the Free Field. The two-dimensional contour map of T0 is shown in Figure 8; the straight line $\left(L_{1}\right)$ marked in this figure is the longitudinal axis line through the center of the data collection zone. Figure 8 shows the following characteristics of Rayleigh wave propagation: the Rayleigh wave diffuses in a radial direction that is centered over the vibration source along the surface of the foundation and shows a trend of continuous dissipation. With increasing distance from the vibration source, the dissipation of the acceleration amplitude becomes larger; the minimum value of $\zeta$ shown in the figure lies between 0.1 and 0.2 , and the area of 0.1 to 0.2 occupies a large portion of the whole area.

Further, a cross section based on $L_{1}$ is used to perform a one-dimensional analysis; the associated changes of $\zeta$ are shown in Figure 9. The name $\alpha$ of the horizontal axis in Figure 9 refers to the distance from a certain point to the vibration source. The dissipation characteristics of the acceleration amplitude of the test points in the zone embodied by the cross section of $L_{1}$ are shown in Table 5 . The rate of decline in the table refers to the ratio of the $\zeta$ difference to the corresponding distance between any two points in the field with the unit of $\mathrm{m}^{-1}$. The explanation of Table 5 is given in conjunction with Figure 9. The propagation characteristics of the Rayleigh wave are mainly reflected in the dissipation of the acceleration amplitudes at each point in the field, and the dissipation procedure consists of four steps: the first step occurs in the range of approximately $0.2 \mathrm{~m}$ around the vibration source, where $\zeta$ rapidly attenuates to approximately 0.6 and the rate of decline is close to $2 / \mathrm{m}$; the second step occurs at approximately $0.2 \mathrm{~m}-0.4 \mathrm{~m}$, where $\zeta$ remains at approximately 0.6 ; the third step occurs at approximately $0.4 \mathrm{~m}-0.7 \mathrm{~m}$, where $\zeta$ decreases from 0.6 to 0.25 and the rate of decline is close to $1.2 / \mathrm{m}$; the fourth step occurs in the area beyond $0.7 \mathrm{~m}$, where $\zeta$ decreased steadily from 0.25 to 0.1 and the rate of decrease rate remains at $0.14 / \mathrm{m}$; the value of $\zeta$ 


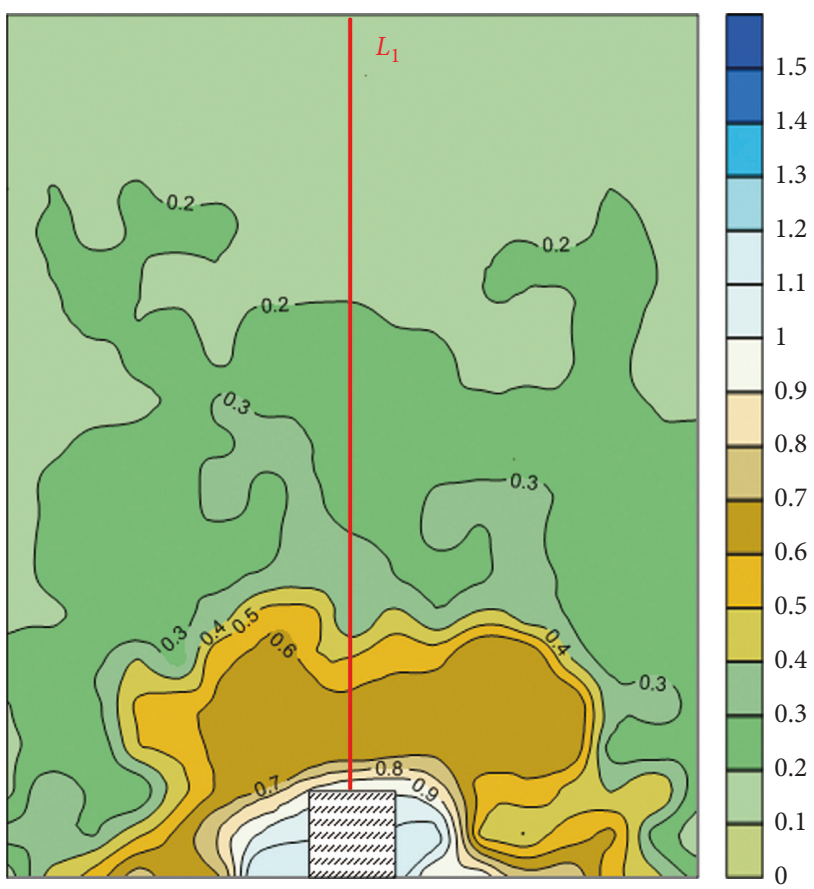

Figure 8: The contour map of $\zeta$ on T0.

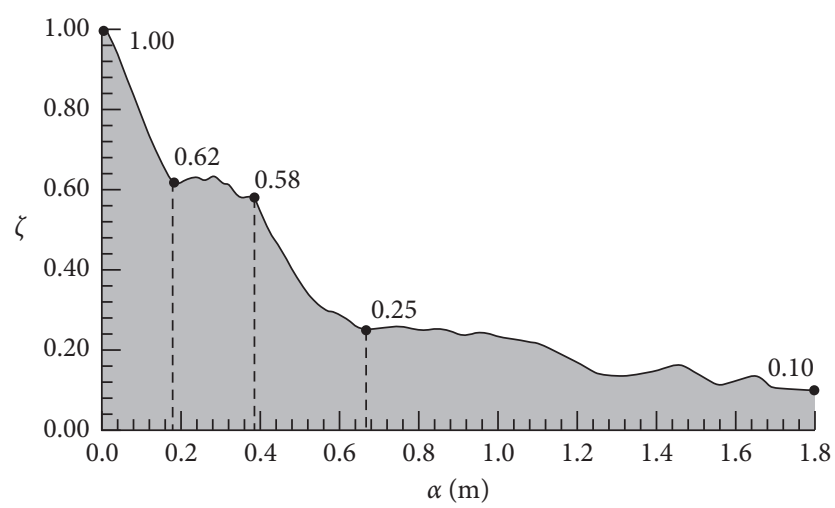

Figure 9: Dissipation characteristics of $\zeta$ along $L_{1}$ on T0.

TABle 5: Dissipation characteristics of $\zeta$.

\begin{tabular}{lccc}
\hline Procedure & Range $(\mathrm{m})$ & Decline rate $\left(\mathrm{m}^{-1}\right)$ & Evaluation \\
\hline Step 1 & $<0.2$ & 2 & Rapid \\
Step 2 & $0.2 \sim 0.4$ & 0 & Steady \\
Step 3 & $0.4 \sim 0.7$ & 1.2 & Neutral \\
Step 4 & $>0.7$ & 0.14 & Placid \\
\hline
\end{tabular}

at infinity will theoretically converge to 0 , but this is beyond the scope of the data collection zone.

3.2. The Test of a Single Pile. T1-35 in Table 3 is selected for the vibration isolation analysis for a single pile, and the twodimensional contour map of $\zeta$ based on this case is shown in Figure 10. The pile is buried on the longitudinal axis line through the center of the date collection zone; the distance from the exciter is $0.3 \mathrm{~m}$; and the pile length is $0.5 \mathrm{~m}$, which

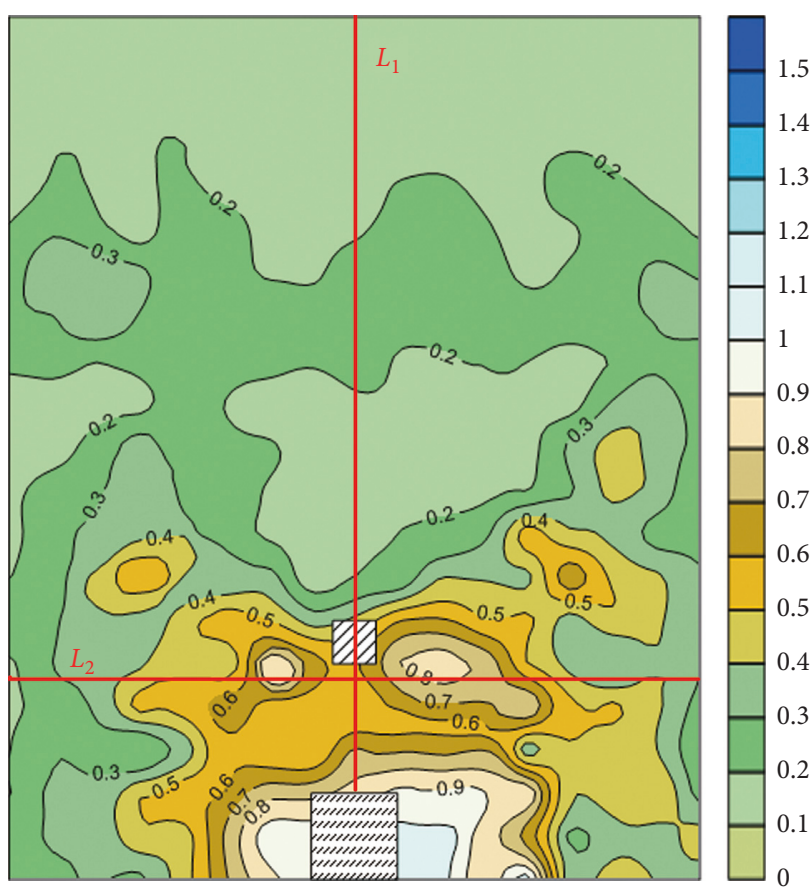

Figure 10: The contour map of $\zeta$ on T1-35.

corresponds to Figure 6(a). The $L_{1}$ marked in Figure 10 is the longitudinal axis through the center of the data collection zone; the $L_{2}$ is located in close proximity to the anterior lateral wall of the pile parallel to the transversal axis of the data collection zone, and both are used for one-dimensional analysis.

Comparing T1-35 with $\mathrm{T} 0$, there are some changes in the contour map of $\zeta$ in the data collection zone that are reflected in the following two aspects. On the one hand, a triangular vibration-shielded region is formed behind the single pile whose $\zeta$ is between 0.1 and 0.2 , and the single pile shows a certain vibration isolation effect. At the same time, it is undeniable that the shielded region of a single pile is small and its isolation effect is limited. On the other hand, there are two obvious vibration-strengthened areas near the two anterior angles of the pile, and two vibration-strengthened strips form along the sides of the shielded region, ranging from the strengthened region to the zone behind the pile. The vibrations of the field in the strengthened region and the strengthened strips are greater than those in the surrounding area, excluding the shielded region. The reason for the formation of the strengthened region and the strengthened strips is that the energy converges from the diffraction at the two sides of the single pile when the Rayleigh wave passes through the pile.

The longitudinal profile along $L_{1}$ in Figure 10 is compared with that at the corresponding position of $\mathrm{T} 0$, and the contrasting results are shown in Figure 11 . Compared with $\mathrm{T} 0$, the position where the $\zeta$ curve begins to enter the placid part is delayed in T1-35. A rebound on the $\zeta$ curve is present in front of the pile, and the value of this rebound is nearly $20 \%$. The vibration-shielded region is covered to $0.5 \mathrm{~m}$ behind the pile. The vibration in the 


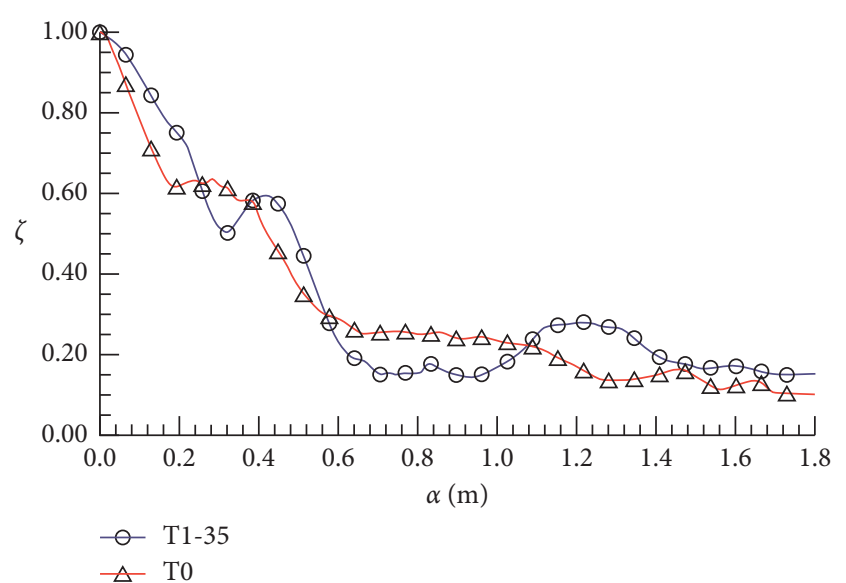

FIGURE 11: Variation of dissipation rate on $L_{1}$ of the single pile test.

shielded region is weaker than that in T0, and the opposite pattern is observed outside the shielded region. Therefore, the action mechanism of the pile on the Rayleigh wave is not simple weakening but rather energy redistributing. As far as the isolation effect, the maximum of which is $42.10 \%$, a distance of $0.74 \mathrm{~m}$ is found between the pile and the point.

The transverse profile along $L_{2}$ in Figure 10 is compared with that of the corresponding position of $\mathrm{T} 0$, and the contrasting results are shown in Figure 12. The variable $\beta$ of the horizontal axis of Figure 12 denotes the shortest distance from a point to the longitudinal axis of the data collection zone, which goes through the center of the zone. As shown in Figure 12, there are two peak values in the $\zeta$ curve of T1-35, which are near 0.9 . The largest percentage increase is $35.35 \%$ higher than that of the corresponding position in T0. The peak values are located on both sides of the pile, which is consistent with the characteristics of the strengthened region near the two anterior angles of the pile in Figure 10. The $\zeta$ value on the lateral sides of the peak decrease rapidly, and the $\zeta$ value remains at the level of T0 when it reaches the edge of the data collection zone. Analyzing the curve of T0, $\zeta$ remains mostly around the level of 0.6 and decreases to 0.2 near the edge of the data collection zone, which is consistent with the characteristics of the second step shown in Figure 9 and Table 5.

3.3. The Test of the Double Piles. T2-353 in Table 3 is selected for the vibration isolation analysis of the double piles, and the two-dimensional contour map of $\zeta$ based on this case is shown in Figure 13. The exciter is aligned to the blank area between the two piles, which is different from the case of T135 . The source distance and pile length are the same as those of T1-35, and a clear distance of $0.3 \mathrm{~m}$ is added, which corresponds to the results shown in Figure 6(b). The $L_{1}$ marked in Figure 13 is the longitudinal axis through the center of the data collection zone; $L_{2}$ is a line of the same length as $L_{1}$, which connects the vibration source and the center of the cross section of the pile; $L_{3}$ is a line running through the data collection zone and connects the centers of the cross section of two piles, which runs parallel to the

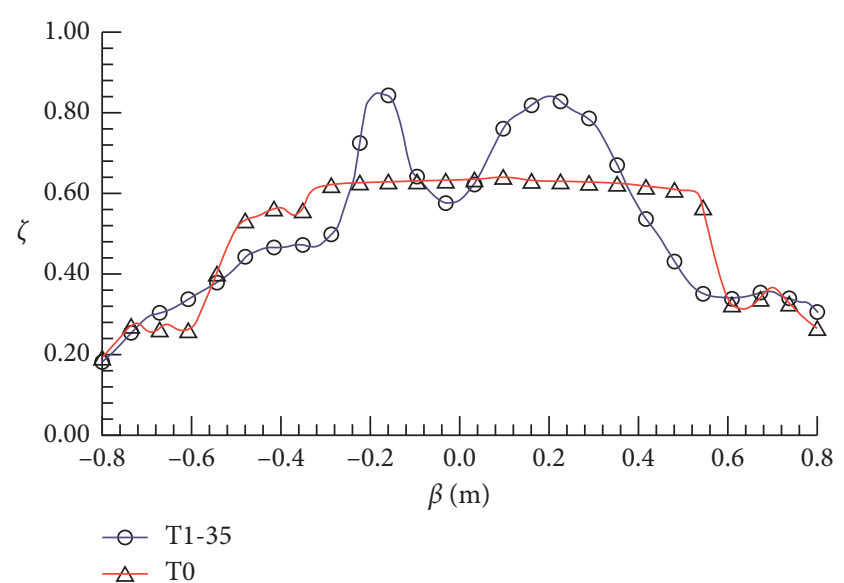

FIGURE 12: Variation of dissipation rate on $L_{2}$ of the single pile test.

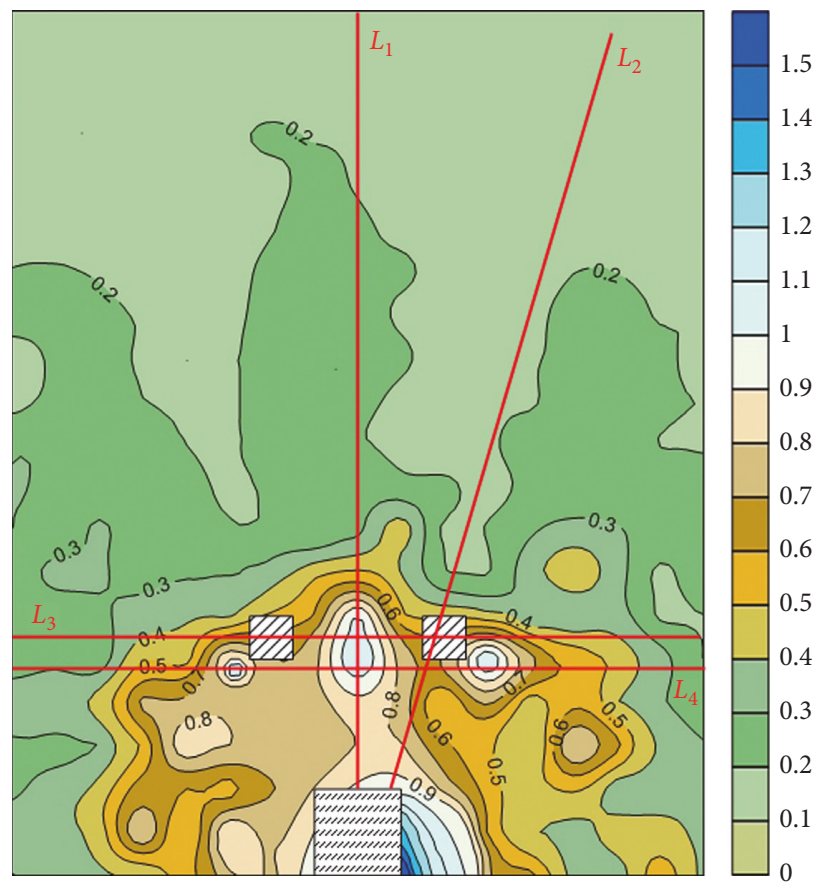

FIGURE 13: The contour map of $\zeta$ on T2-353.

transverse axis of the data collection zone; $L_{4}$ is located in close proximity to the anterior lateral wall of the pile parallel to $L_{3}$.

Compared with $\mathrm{T} 0$, the configuration of the pile in T2353 has the same two effects on the $\zeta$ 's two-dimensional contour map. The first is the shielded effect, and two trumpet-shaped vibration-shielded regions are formed behind the pile. With increasing distance from the pile, the opening of the shielded region increases gradually. The second effect is the enhancement effect, and three vibrationstrengthened regions are formed due to different causes in the gap between the piles and on both sides of the pile angle. One of the strengthened regions is located in the gap of the piles; the energy convergence of the reflection of Rayleigh waves on both sidewalls of the piles leads to the vibration being strengthened. The energy convergence of the reflection 
of Rayleigh waves occurs on both sidewalls of the piles, leading to strengthened vibrations. The Rayleigh wave is scattered through the gap of the piles, and the scattering wave continues to propagate into the postpile region. The other two strengthened regions have similar causes of formation to those in the test of a single pile and are located near the two anterior lateral angles of the pile. Similarly, this phenomenon involves the diffraction of Rayleigh waves.

Compared with the single pile test of T1-35, the double pile test of T2-353 shows some new characteristics. First, there are two shielded regions corresponding to the number of piles whose shapes are not "closed triangles" but "open trumpets." The shielded region with a $\zeta$ value of 0.1-0.2 runs through the region with $\zeta$ values of 0.2 to 0.3 . The area of the shielded region increases, and the isolation effect of the test of double piles is better than that of a single pile. There is no especially obvious vibration-strengthened strip like that found in the test of a single pile. This is additional proof that the isolation effect of the double piles is better than that of the single pile. In terms of the shielded region, T2-353 shows some of the same characteristics as T1-35. First, the shape of the shielded region is the same, and the shielded effect determines the opening or closing state of the region. Second, in the data classification method of the $\zeta$ values shown in Figure 13, the shielded region mainly affects the zone of $\zeta$ value between 0.2 and 0.3 , which is mainly reflected in the area but has little effect on the other regions.

For further analysis, for the longitudinal sections of $L_{1}$ and $L_{2}$ in Figure 13 and compared with the $L_{1}$ profile of T0, the variation of $\zeta$ is shown in Figure 14. T2-353: $L_{1}$ passes through the gap of the piles, and the strengthened region is located at the above position, which is reflected in Figure 14 as a rebound of the $\zeta$ value at $0.4 \mathrm{~m}$. The peak value of $\zeta$ is 1.15 times greater than the $\zeta$ of the vibration source. After the peak value, $\zeta$ decays sharply and tends to be flat at $0.6 \mathrm{~m}$. T2-353; $L_{1}$ is greater than T0; and on the whole, $L_{1}$ shows remarkable strengthening. The trend of the curve of T2-353 shows that $L_{2}$ is similar to the test of the free foundation, but the position where the curve begins to flatten is earlier than the $0.6 \mathrm{~m}$ of T0: $L_{1}$. The isolation effect in the shielded region of the test of double piles is better than that in the test of a free foundation. Figure 15 shows a comparison between T2353 and T0 at the position of $L_{3}$ in Figure 13. The line connects the centers of the cross sections of two piles. The figure shows that the $\zeta$ curve of T2-353 is higher than that of $\mathrm{T} 0$, and the average percentage increase of $\zeta$ is $39.46 \%$. The $\zeta$ peak of T2-353, which is close to 1.1, appears in the gap of the double piles. The peak of T2-353 is 3 times as high as the $\zeta$ value of T0 in the corresponding position. Figure 16 shows a comparison between T2-353 and T0 at the position of $L_{4}$ in Figure 13. The two curves in Figure 16 basically coincide at the edges of both sides, and the difference occurs in the middle of the curve. There are three peaks on the T2-353 curve, which are located in the gap and on the outsides of the double piles. The middle peak corresponds to the scatteringstrengthened region in Figure 13, and the peaks on both sides correspond to the strengthened regions of diffraction in Figure 13. Three peaks of the $\zeta$ curve are all close to 1.1, an increase of nearly $66 \%$ compared with that of $\mathrm{T} 0$.

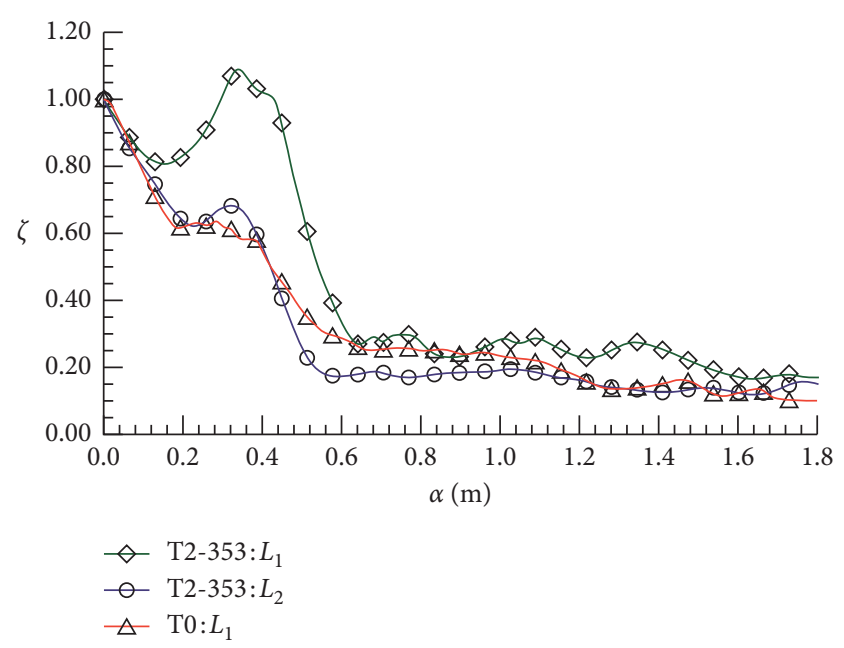

FIGURE 14: Variation of the dissipation rate on $L_{1} / L_{2}$ of the double pile test.

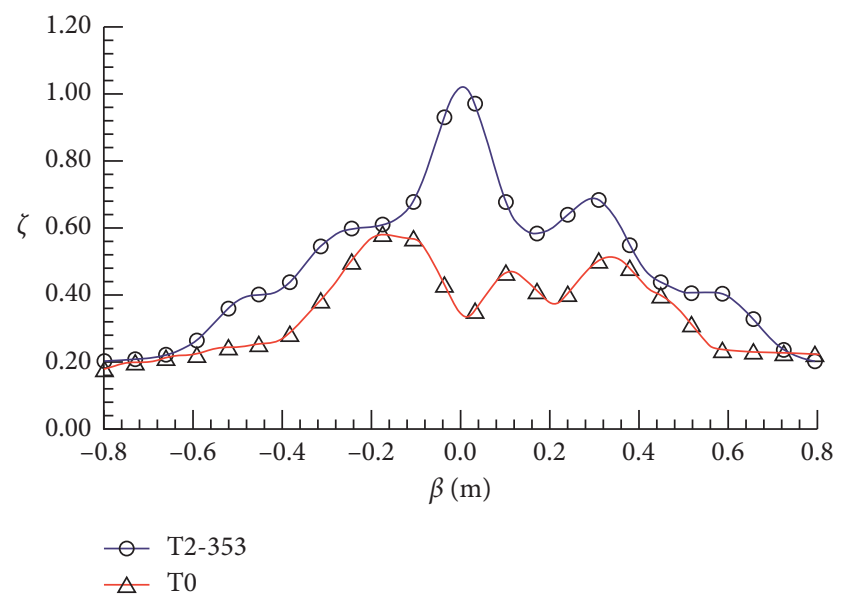

FIgURE 15: Variation of the dissipation rate on $L_{3}$ of the double pile test.

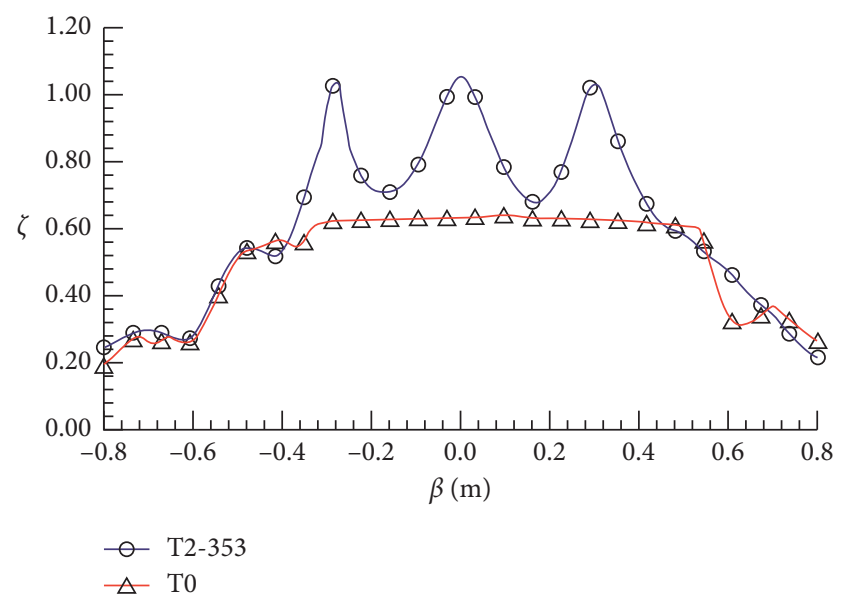

FIGURE 16: Variation of the dissipation rate on $L_{4}$ of the double pile test. 
3.4. The Test of the Treble Piles. T3-353 in Table 3 is selected for the vibration isolation analysis of the treble piles, and the two-dimensional contour map of $\zeta$ based on this case is shown in Figure 17. One pile was added to each side with a clear distance of $30 \mathrm{~cm}$ on the basis of T1-35. The source distance and pile length are the same as those in T1-35, which corresponds to Figure 6(c). The $L_{1}$ marked in Figure 17 is the longitudinal axis through the center of the data collection zone; $L_{2}$ is a line of the same length as $L_{1}$, which connects the gap midpoint of the two piles and the vibration source; $L_{3}$ is a line running through the data collection zone and connects the centers of the cross section of the three piles, which is parallel to the transverse axis of the data collection zone; $L_{4}$ parallel to $L_{3}$ is located in close proximity to the midpoint of the middle pile and the vibration source.

T3-353 differs from the T1-35 test in the following two aspects: (1) the region of $\zeta<0.1$ appears behind the piles and has a symmetrical shape that was not seen in the previous tests. At the same time, the region of $\zeta<0.2$ is larger than that of T1-35. Therefore, increasing the number of piles is helpful to improving the isolation effect in the postpile area. (2) There is a remarkably strengthened region in front of the piles, which is called the composite region and lies between the exciter and the middle pile; the $\zeta$ peak value in the center of the region reaches more than 1.5 , which is higher than the intensity of the vibration source. This region does not appear in $\mathrm{T} 0$ and has a certain representation in T1-35 but neither case reached the level of T3-353. The reason for this strengthened region is probably the strong vibration superposition of the two scattering-strengthened regions in the gap of the piles and the two diffraction-strengthened regions near the anterior angles of the piles. Because of the small quantity of piles, the superposition effect of the strengthened region is weak, which leads to the region being not very significant in T1-35. According to the above analysis, increasing the quantity of piles has two effects on the surface vibration of the foundation. On the one hand, it can enhance the isolation effect of the shielded region and further reduce the surface vibration behind the piles; on the other hand, it inevitably amplifies the surface vibration of the strengthened region, especially in the area in front of piles.

Compared with T2-353, T3-353 has the following similar characteristics. (1) There are two gaps among the treble piles, and two scattering-strengthened regions corresponding to the two gaps are located in the gaps. In addition, there are two strengthened strips behind the piles connected to the scattering-strengthened regions, which are much more obvious than that of T2-353. (2) Three shielded regions are formed along the line linked to the vibration source and piles. Limited to the direction of the data collection zone and the relative position of the piles and the vibration source, the shielded region behind the middle pile is more obvious than the other two regions. (3) Two diffraction-strengthened regions similar to T2-353 are formed at the anterior angles of the row pile, and there is also no strengthened strip similar to that of T1-35 behind the strengthened region.

The comparison of T3-353 and T0 in the onedimensional analysis is shown in Figures 18-21, and each figure corresponds to the sections of $L_{1} \sim L_{4}$. Figure 18 shows

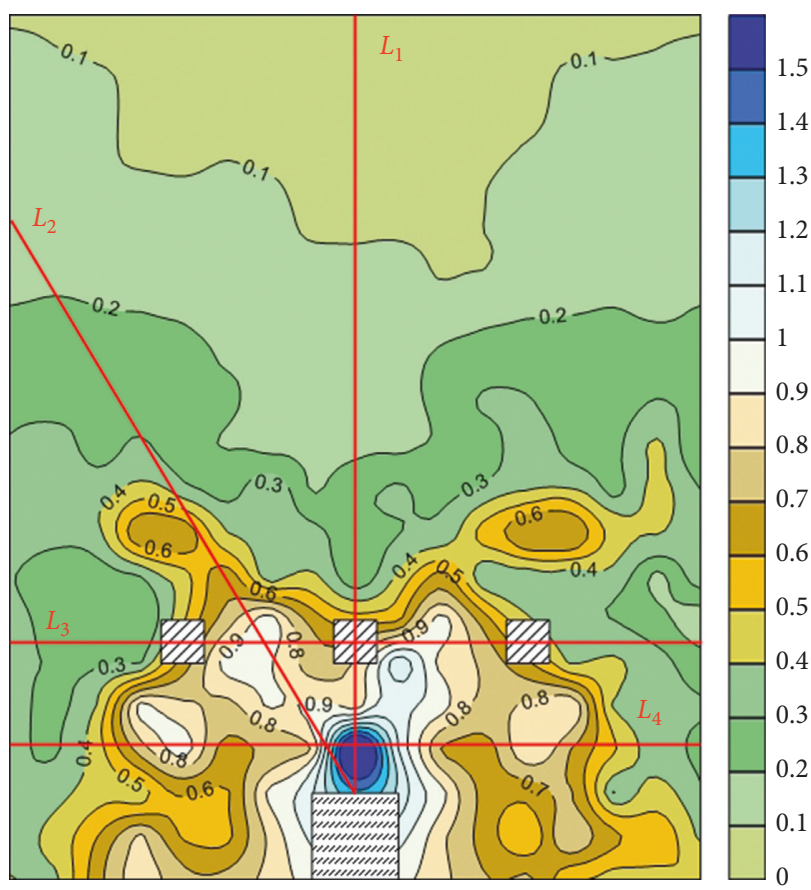

Figure 17: The contour map of $\zeta$ on T3-353.

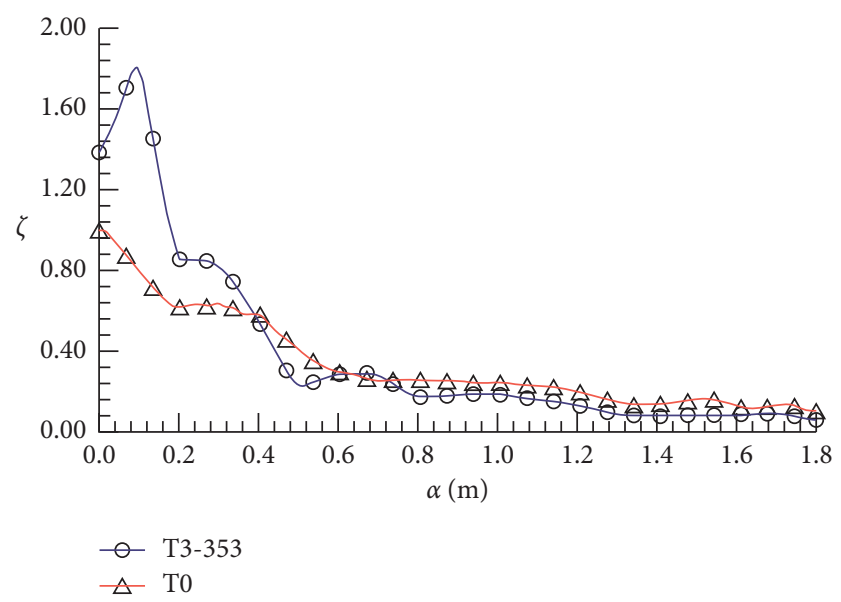

FIgURE 18: Variation of dissipation rates on $L_{1}$ of the treble pile test.

the comparison between T3-353 and T0 on $L_{1}$. The figure shows that the two curves are divided into two stages with the pile as the dividing line. T3-353 is greater than T0 in the front of the piles and is exactly the opposite behind the piles, which is consistent with the analysis of the $L_{2}$ of T2-353 in Figure 13. Specifically, for data analysis, the maximum percentage increase in front of the pile was $124.84 \%$, which is beyond the levels of T1-35 and T2-353. The vibration of the foundation surface of T3-353 behind the piles is generally lower than that of $\mathrm{T} 0$, and the result of data analysis shows that the average decrease between the two is $30.54 \%$. In particular, the $\zeta$ value of T3-353 is less than 0.1 in beyond $1 \mathrm{~m}$ behind the piles, which is close to $50 \%$ lower than that of T0, and the isolation effect is quite remarkable. Figure 19 shows a comparison between T3-353 and T0 on $L_{2}$. As shown in the figure, the vibration of the foundation surface 


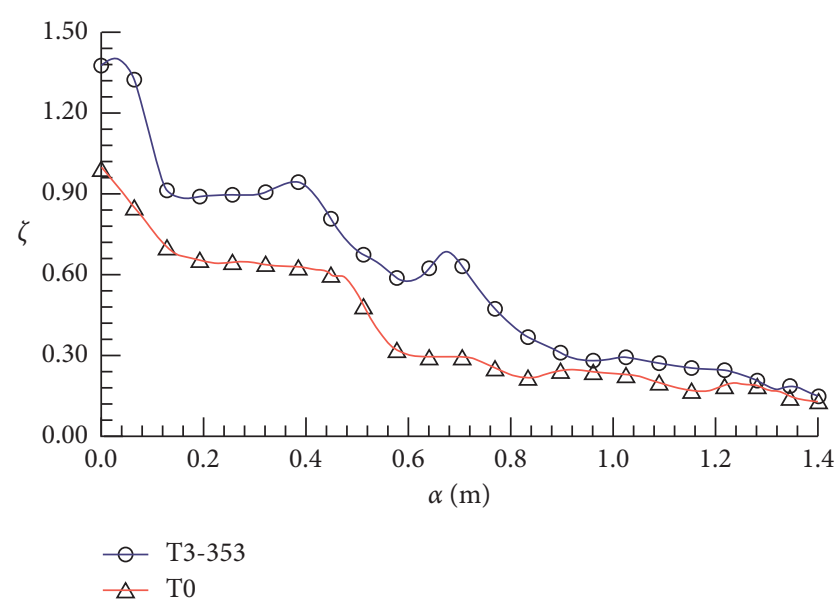

FIGURE 19: Variation of dissipation rates on $L_{2}$ of the treble pile test.

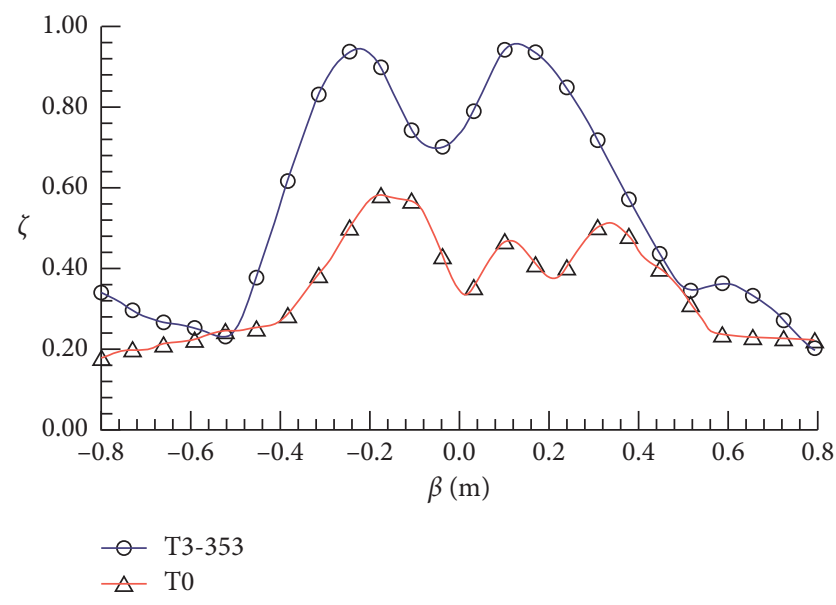

FIGURE 20: Variation of dissipation rates on $L_{3}$ of the treble pile test.

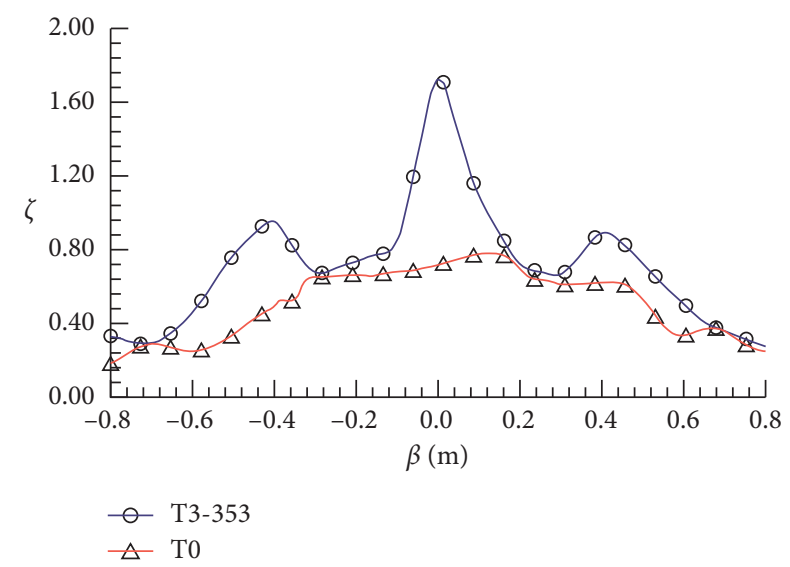

Figure 21: Variation of dissipation rates on $L_{4}$ of the treble pile test.

of T3-353 on the line linking the vibration source to the gap between piles is generally greater than that of $\mathrm{T} 0$, which is consistent with the characteristics of the strengthened strips in Figure 17. With increasing distance from the vibration source, the two curves in Figure 19 tend to coincide behind the piles, and the influence of the scattering wave tends to weaken. Figure 20 shows a comparison between T3-353 and T0 on $L_{3}$. As shown in the figure, the $\zeta$ curve of T3-353 is generally greater than T0. The peak of T2-353 is close to 1.1, appears in the gaps between neighboring piles, and is 0.6 greater than that of T0. The maximum increase of $\zeta$ at the same positions at T3-353 to T0 is $140.41 \%$, and the strengthened vibrations in the gaps of the neighboring piles are obvious. Figure 21 shows the vibration of the foundation surface in front of the row piles for a comparison of T3-353 with T0. $L_{4}$ runs parallel to $L_{3}$ and is located in close proximity to the midpoint of the middle pile and the vibration source, crossing the center of the diffraction vibration region at the anterior angles of the two sides of the piles. There are three peaks in T3-353 corresponding to the two diffraction-strengthened regions and the composite strengthened region, respectively. The middle peak is 1.81 times and 1.94 times the peaks on both sides, respectively. Because $L_{4}$ does not actually pass through the core of the composite strengthened region, the $\zeta$ peak value at the center of the composite strengthened region is likely to be higher. In addition, the value of the T3-353 curve is obviously greater than that of the T0 test; the two curves are only close to each other at the edges of both sides, and the average increase is $44.15 \%$. The vibration of the foundation surface in front of the piles is greatly strengthened by the row pile.

\section{Conclusions}

Based on the field measurements, the two-dimensional contour map of the acceleration amplitude dissipation rates of the vibration source is drawn using a vibration test carried on a sand foundation. In addition, the means of the one-dimensional linear analysis of the characteristic sections on the contour map are studied. The propagation characteristics of the Rayleigh wave and the vibration isolation mechanism of single-row of piles on the surface of the sand foundation are analysed. Under the experimental conditions of this paper, the results are as follows:

(1) The Rayleigh wave diffuses radially around the vibration source in the test of the free field and tends to decay with increasing propagation distances. Specifically, there are four steps and two node values in the dissipative process of the Rayleigh wave with $\zeta$ as the reference index. There will be a steady transition when $\zeta$ drops to 0.6 and a placid decline when $\zeta$ is less than 0.25 ; the decay in the other steps will be fast.

(2) The effect of the single-row of piles on the vibration of the foundation surface is shown in two aspects: the shielding effect behind the piles and the strengthening effect in front of the piles.

(3) The vibration-shielded region is located behind the piles, and the region presents a trumpet-shaped area that uses the pile as the vertex. Increasing the quantity of piles contributes to increasing the vibration isolation effect not only by involving the degree of isolation but also the area of the shielded region. The shielding effect behind the piles is mainly caused by the transmission of the wave. 
(4) Some regions are distributed in front of and to the rear of the piles, and the gaps between the piles show strengthened effects. The phenomenon of vibrationstrengthening relates to the vibration-strengthened regions and the vibration-strengthened strips. The strengthened regions are divided into three types according to their cause of formation. The first is formed by the diffraction of a Rayleigh wave, which occurs at the anterior angles of the two sides of the row pile. The second is formed by the scattering of a Rayleigh wave, which occurs in the gaps between the neighboring piles. The third type occurs between the vibration source and the piles and is caused by the superposition of the vibrations among the diffraction-strengthened regions and the scatteringstrengthened regions and the vibration source. The analysis shows that the more piles there are, the more obvious the vibration strengthening is. In general, the vibration-strengthened strips of the single-row of piles with arbitrary numbers of piles are connected with the scattering-strengthened regions, which start in the gaps and extend to a certain range behind the pile. The quantity of the vibration-strengthened strips corresponds to the quantity of the gaps between piles, and the ranges of the strips differ. In particular, there are two vibration-strengthened strips in the test of a single pile, which are connected with the diffraction vibration-strengthened region and adjacent to the vibration-shielded region.

\section{Data Availability}

The data used to support the findings of this study are available from the corresponding author upon request.

\section{Conflicts of Interest}

The authors declare that there are no conflicts of interest regarding the publication of this paper.

\section{Acknowledgments}

The authors thank American Journal Experts Inc. for providing the English editing service. This paper was supported by the Youth Talent Projects of Colleges in Hebei Province of China (No. BJ2016018). In addition, the Scientific and Technological Program of Research and Development of Zhangjiakou (1811009B-13) also provided financial support for this paper.

\section{References}

[1] G. Shi and G. Y. Gao, "Three dimensional analysis of a row of piles as passive barriers in saturated soil," Journal of Vibration Engineering, vol. 23, no. 5, pp. 546-553, 2010.

[2] J. L. Liu, G. S. Feng, R. H. Zhang et al., "Orthogonal test on subway vibration isolation of single rank piles adjacent to existing building," Railway Standard Design, vol. 61, no. 12, pp. 126-130, 2017.
[3] H. Z. Wang, "Analysis on the factors of potential environmental hazards in Longmen Grottoes of Luoyang City," Military Investigation, vol. 8, no. 4, pp. 44-47, 1994.

[4] X. J. Yang, "Soil dynamics in industrial environment vibration," Chinese Journal of Geotechnical Engineering, vol. 14, no. 2, pp. 82-88, 1992.

[5] X. J. Yang and Y. Q. Mao, "A discussion on ground vibration characteristics and attenuation caused by traffic vehicles," Journal of Building Structures, vol. 8, no. 5, pp. 77-80, 1987.

[6] R. D. Woods, "Screening of surface waves in soils," Journal of the Soil Mechanics and Foundation Division, vol. 94, no. 4, pp. 951-979, 1968.

[7] G. S. Feng, J. L. Liu, R. H. Zhang et al., "Experimental research for influence of filling properties on vibration isolation effect of sand-filled trench," Railway Engineering, vol. 57, no. 2, pp. 151-155, 2017.

[8] L. G. Zhang, J. L. Liu, X. G. Song et al., "The numerical analysis of effect and influencing factor of vibration reduction by open trench on high-speed railway," Journal of Railway Science and Engineering, vol. 14, no. 7, pp. 1354-1360, 2017.

[9] G. Shi and G. Y. Gao, "Semi-analytical boundary element method in saturated soil and its application to analysis of double row of piles as passive barriers," Rock and Soil Mechanics, vol. 31, no. 2, pp. 59-64, 2010.

[10] E. P. Hou, J. L. Liu, R. H. Zhang et al., "Model test study on vibration isolation effect of concrete piles in rows for railway subgrade," Railway Engineering, vol. 57, no. 3, pp. 141-145, 2017.

[11] J. Aviles and F. J. Sanchez-Sesma, "Piles as barries for elastic waves," Journal of Geotechnical Engineering Division, vol. 109, no. 9, pp. 1133-1146, 1983.

[12] R. D. Woods, N. E. Barnet, and R. Sangesser, "A new tool for soil screening of surface waves in soil dynamics," Journal of Geotechnical Engineering Division, vol. 100, no. 11, pp. 1234-1247, 1974.

[13] S. E. Kattis, D. Polyzos, and D. E. Beskos, "Modelling of pile wave barriers by effective trenches and their screening effectiveness," Journal of Soil Dynamics and Earthquake Engineering, vol. 18, no. 1, pp. 1-10, 1999.

[14] S. E. Kattis, D. Polyzos, and D. E. Beskos, "Vibration isolation by a row of piles using a 3-D frequency domain BEM," International Journal for Numerical Methods in Engineering, vol. 46, no. 5, pp. 713-728, 1999.

[15] M. Q. Xu, "Analysis of passive isolation of vibration due to moving loads using pile rows embedded in a poroelastic half space," Rock and Soil Mechanics, vol. 31, no. 12, pp. 39974005, 2010.

[16] Z. X. Liu and S. J. Wang, "Isolation effect of discontinuous pile-group barriers on plane $\mathrm{P}$ and SV waves: simulation based on two-dimensional broadband indirect boundary integration equation method," Rock and Soil Mechanics, vol. 37, no. 4, pp. 1195-1207, 2016.

[17] M. M. Sun, "Multiple scattering of $\mathrm{SH}$ waves by rows of arbitrarily arranged tubular piles," Rock and Soil Mechanics, vol. 35, no. 4, pp. 943-950, 2014.

[18] P. Xu, D. M. Yan, Y. H. Deng et al., "Isolation of elastic waves by discontinuous barrier composed of a row of rigid piles," Journal of Vibration and Shock, vol. 26, no. 11, pp. 133-137, 2007.

[19] J. T. Kenney, J. R. Pasadina, and Galif, "Steady-state vibrations of beam on elastic foundation for moving load," Journal of Applied Mechanics, vol. 21, pp. 359-364, 1954. 
[20] M. L. Baron and A. T. Matthews, "Diffraction of a pressure wave by a cylindrical cavity in an elastic medium," Journal of Applied Mechanics, vol. 83, no. 3, pp. 347-354, 1961.

[21] A. K. Mal and L. Knopoff, "Transmission of Rayleigh waves past a step change in elevation," Bulletin of the Seismological Society of American, vol. 55, no. 2, pp. 319-334, 1965.

[22] V. V. Tyutekin, "Scattering of plane waves by a cylindrical cavity in an isotropic elastic medium," Soviet PhysicsAcoustics, vol. 5, pp. 105-109, 1959.

[23] I. A. Viktorov, "The effects of surface defects on the propagation of Rayleigh waves," Soviet Physics-Doklady, vol. 3, pp. 304-306, 1958.

[24] G. F. Miller and H. Pursey, "On the partition of energy between elastic waves in a semi-infinite solid," Proceedings of the Royal Society of London, vol. 233, no. 1192, pp. 55-59, 1955. 


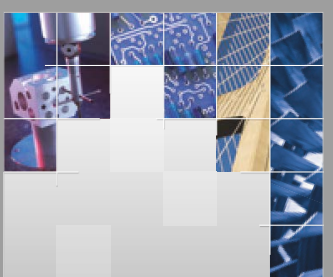

\section{Enfincering}
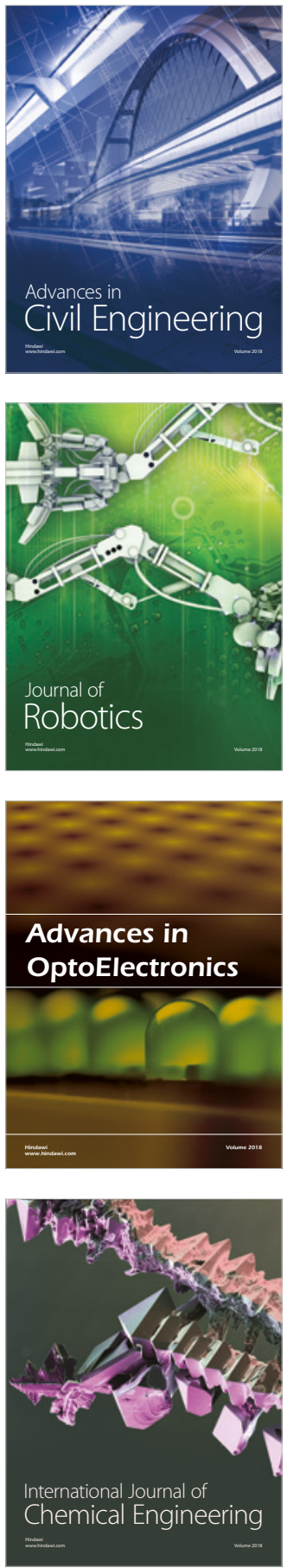

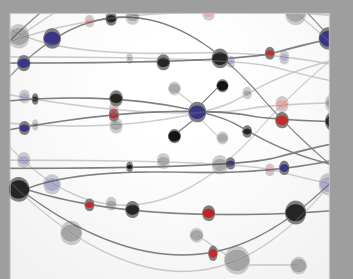

\section{Rotating \\ Machinery}

The Scientific World Journal

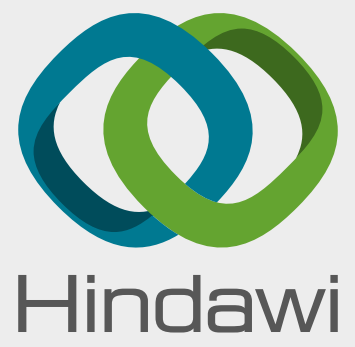

Submit your manuscripts at

www.hindawi.com
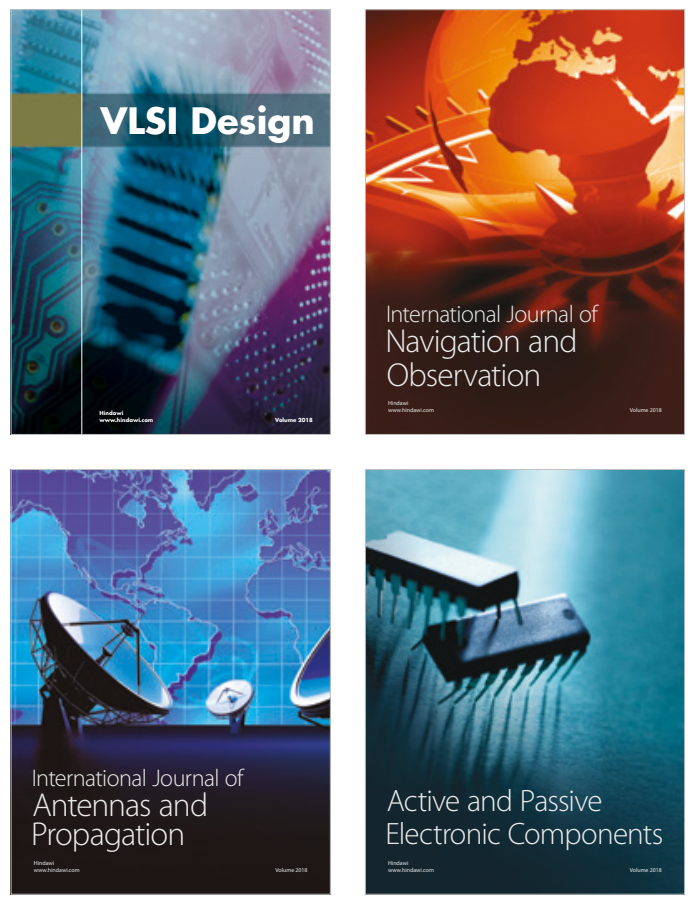
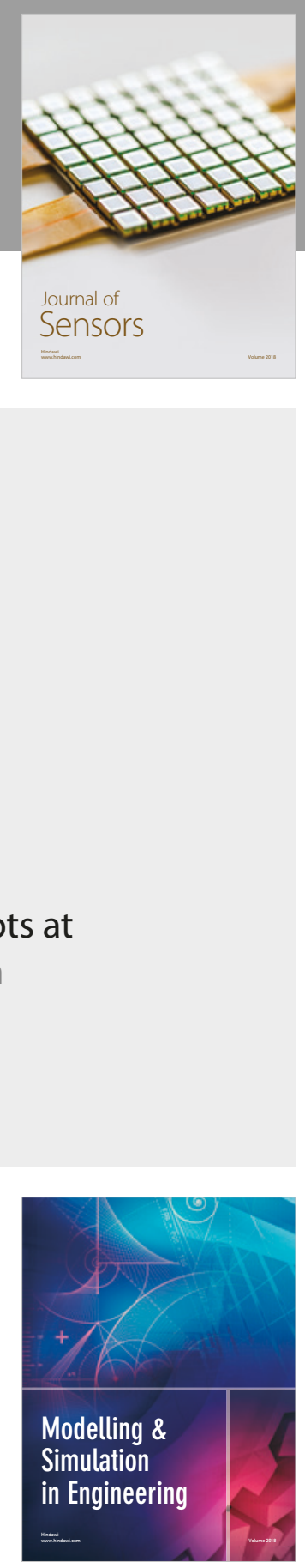

\section{Advances \\ Multimedia}
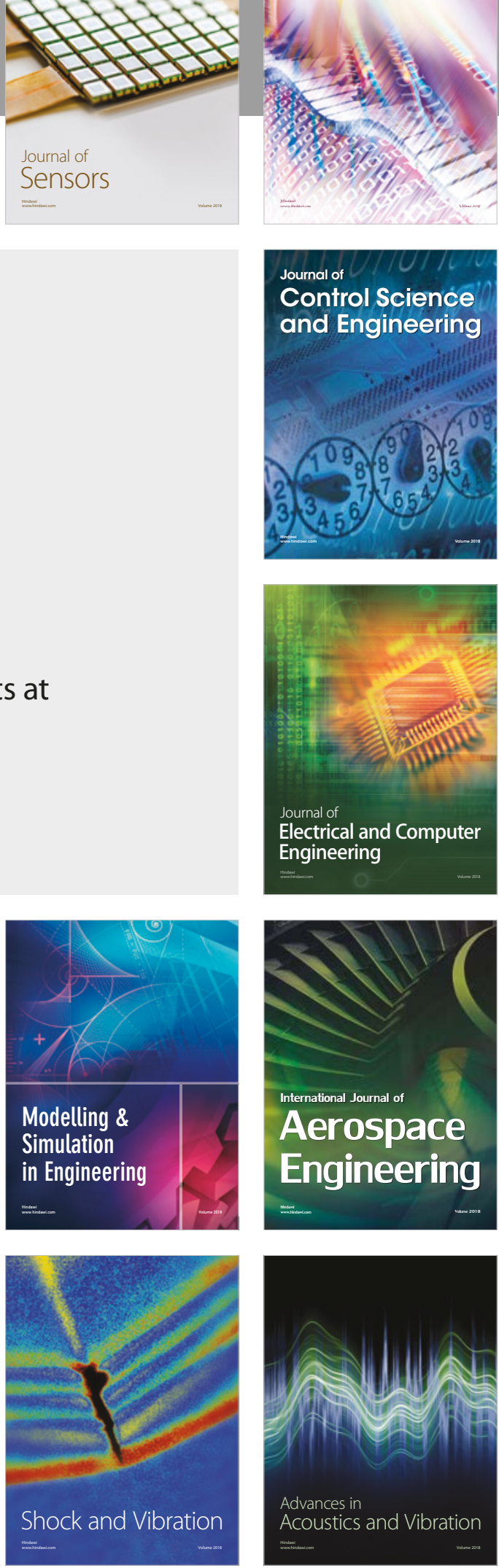